\title{
Molecules
}

\section{Oriented by Brute Force}

\section{H.J. Loesch}

Fakultat fur Physik, Universität Bielefeld, D-33501 Bielefeld

J. Bulthuis and S. Stolte

Laser Centre, Department of Physical and Theoretical Chemistry, Vrij Universiteit, de Boelelaan 1083, NL-1081 HV Amsterdam

\section{A. Durand, J.C. Loison and J.Vigué}

Laboratoire Collisions Agrégats Reactivité, IRSAMC, Université Paul Sabatier and CNRS URA 770

118, route de Narbonne, F-31062 Toulouse Cedex

\section{The spectroscopy and reaction chemistry of molecules with large dipole moments and small rotational constants that have undergone intense rotational cooling before being introduced into an electric field illustrate that manipulation of the rotational degree of freedom of molecules offers an intriguing challenge.}

Techniques to control the orientation of a molecular axis by applying an external field have been sought for many years, because oriented molecules can be used to study the effect on a chemical reaction of the relative orientation of collision partners. The spectroscopy of oriented molecules is also very interesting since free rotational motion is replaced by oscillation over a limited angular range about the field direction ("libration").

The state-selection and orientation of polar molecules goes back to the celebrated Stern-Gerlach experiment in which a double-peaked diffraction pattern was observed on transmitting a beam of silver atoms through an inhomogeneous magnetic field. Each silver atom exhibited an individual Zeeman effect depending on the angular momentum quantum number, and correlation effects between the silver atoms could be ignored. Orienting a molecule (i.e., aligning preferentially one of the molecule's ends along a vector fixed in space) means favouring the pointing of its permanent electric dipole moment parallel or antiparallel to an applied electric field. This orientation effect was investigated in the early days of quantum mechanics by measuring the deflection of polar molecules such as $\mathrm{HCl}$ in an inhomogeneous electric field. Although strong fields of the order of $4 \mathrm{MV} / \mathrm{m}$ were used, the observed deflection was surprisingly small.

This unexpected behaviour can be traced to deviations from the SternGerlach experiment. First, the dipole moment lies along the molecular axis and not, like the magnetic moment, along the angular momentum, so the Stark energy splittings are quadratic in the electric field. Second, in a hot molecular beam, many of the angular momentum quantum rotational sublevels are populated, and each behaves differently.

Subsequent electric deflection experiments investigated the Stark effect of single states, and were not aimed at obtaining an oriented beam. The problem was revived by Paul and coworkers in 1964 who used electric multipolar fields to focus state-selected polar molecules. Kramer and Bernstein [1] pointed out that this method could be used to produce oriented molecular beams of polar symmetric "spinning-top" molecules (e.g., $\left.\mathrm{CH}_{3} \mathrm{I}\right)$, and experiments investigating the effect of molecular orientation on reactive scattering followed shortly, although a quantitative interpretation of the observed orientation effects came later [2].

The method of orienting molecules by applying an electric field was known to be inefficient, notably for molecules such as $\mathrm{HCl}[3]$. Reliable methods to produce oriented molecules by simply applying a strong electric field to a molecular beam, which has undergone intense rotational cooling owing to supersonic expansion, had to await the recent pioneering work by

\begin{tabular}{lllll} 
molecule & ICI & KCI & HF & $(\mathrm{HCN})_{3}$ \\
\hline$D$ & 1.24 & 10.24 & 1.874 & 10.6 \\
$B$ & 0.114 & 0.129 & 20.96 & 0.0156 \\
$\omega$ & 18.3 & 133.3 & 0.15 & 1142
\end{tabular}

Table. Values of the dipole moment $D$ (in Debye) and of the rotational constant $B$ (in $\mathrm{cm}^{-1}$ ) for typical molecules. The calculated $\omega$-value corresponding to a field $\varepsilon=10 \mathrm{MV} / \mathrm{m}$ is also given.
Loesch and Remscheid [4] and by Friedrich and Herschbach [5]. As the basic equations have been available for 60 years, the pendular states which arise in these "brute force" experiments are well understood. Many groups are now using brute-force oriented molecules for reaction dynamics, photodissociation and spectroscopic studies.

\section{The Orientation Process}

Electric fields in a macroscopic volume rarely exceed $100 \mathrm{MV} / \mathrm{m}$, and values of the order of $10-20 \mathrm{MV} / \mathrm{m}$ are typical. Such fields are too weak to modify noticeably the electronic or vibrational structures of most small molecules; but they can strongly modify the rotational motion. For simplicity, consider a linear polar molecule with no net angular momentum other than that due to rotation (i.e., the situation for most molecules in their ${ }^{1} \Sigma$ ground state). The angular momentum $J$ is specified by two quantum numbers, $J$ and $M$, the latter determining the projection of $J$ on an axis fixed in space; $J$ is perpendicular to the molecular axis. In an electric field, the molecule does not rotate freely owing to the interaction of the dipole $D$ along the molecular axis with the field $\boldsymbol{\varepsilon}$. The Hamiltonian for a rotating dipole in an electric field is $\mathcal{H}=B J^{2}-D \varepsilon \cos v$, where $v$ is the angle between $D$ and $\boldsymbol{\varepsilon}$, and $B$ is the rotational constant, which is inversely proportional to the moment of inertia. The eigenstates defined by this Hamiltonian are no longer the free-rotor states $|J, M\rangle$. The interaction term mixes states $|J, M\rangle$ with states $|J \pm 1, M\rangle,(M$ is thus conserved) and when $D \varepsilon$ is comparable to, or larger than, the rotational energy splitting $-2(J+1) B$ to the next higher energy level - strong mixing occurs. The largest effects arise for low-J levels, and the degree of mixing of the parent (or zero-field) state $|J, M\rangle$ with states $|J, M\rangle$ is determined by the dimensionless parameter $\omega=D \varepsilon / B$. The table gives the values of the rotational constant $B$ and of the dipole $D$ for a few polar molecules as well as the $\omega$-value corresponding to a field $\varepsilon$ of $10 \mathrm{MV} / \mathrm{m}$.

Since the mixing dampens out progressively with increasing $|J-J|$, the energy levels and the associated wavefunctions can easily be calculated by numerical diagonalization of the Hamiltonian matrix on a truncated basis set of free-rotor functions $[4,6]$. Many analytical results can also be established using perturbation theory [7]. The eigenfunctions provide the angular distribution function of the dipole rotors, 


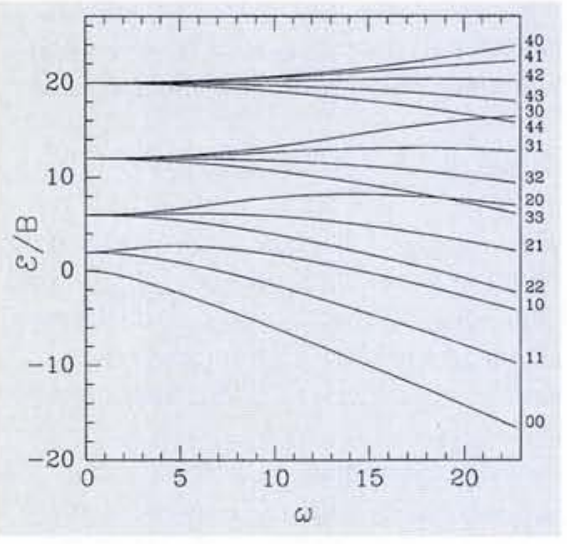

Fig. 1. The energies of the lowest $J M$ levels as a function of the dimensionless parameter $\omega$. The levels are labelled 00 , 11 , etc. according to their quantum numbers $J, M$ defined at zero field. The energy unit is the rotational constant $B$. At high $\varepsilon$-values the interaction with the field is so strong that the dipoles no longer spin freely.

from which one deduces the average orientation $\langle\cos v\rangle$.

Fig. 1 presents the energies of the lowest angular momentum levels as a function of the dimensionless parameter $\omega$. The levels are labelled by $J$ and $M$ of the corresponding zero-field state. At high $\omega$ values the interaction with the field is so strong that the dipoles no longer rotate; in the lowest levels they make only small excur-sions around the field direction, $v \cong$ $\mathrm{o}$, and their motion is well-described by a two-dimensional spherical oscillator. Consequently, the orientation $\langle\cos v\rangle$ of these states is close to 1 . Friedrich and Herschbach [5] coined the term "pendular states" to characterise this restrained angular motion of the dipole (these states were also called "librational states" and the motion "hindered rotation").

At small $\omega$-values, each $J$-level (except $J=0$ ) is split and molecules in low $M$-levels have a negative orientation. This behaviour is most striking for $M=0$, where it is comparable with the classical motion of a hindered rotor. If the dipole has just enough energy to still make full rotations, its angular velocity is considerably larger when $v=0$ than when $v=\pi$. Hence, the dipole resides for a longer time in the antiparallel direction than in the parallel direction and $\langle\cos v\rangle\langle 0$. In a molecular beam, the $M$-averaged rather than the $M$-specific orientation is relevant. For low $\omega$-values, say $\omega<10-20$, the $M$-averaged orientation is noticeable only for very low $J$-levels $(J<5$; see Fig. 2). It is therefore very important to produce a rotationally very cold molecular beam $\left(T_{\text {rot }}<15 \mathrm{~K}\right)$ in order

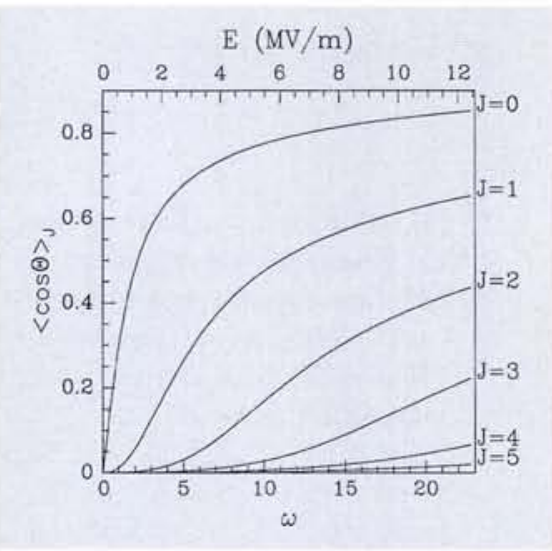

Fig. 2. Plot of the $M$-averaged orientation as a function of $\omega$ for the level $J=0-5$. Only the lowest $J$-levels contribute significantly to the orientation for modest $\omega$-values. It is thus important to produce a rotationally very cold molecular beam so as to obtain an appreciable orientation effect. This is the principle of the brute-force method.

to obtain an appreciable orientation effect. This is the principle of the so-called "brute force" method of orienting molecules, where for systems with large dipole moments and small rotational constants, electric fields which can be realised in practice ensure orientation by virtue of the fact that the electric dipole experiences a significant torque.

The angular distribution of the dipole averaged over a thermal distribution of parent rotational levels is given approximately by

$$
W(v)=\left[1+A_{\mathrm{o}} \cos v\right] / 2
$$

provided $\mathrm{B}<<\mathrm{k} T_{\text {rot }}$ and $\omega$ is not too large $(\omega<10)$, The anisotropy parameter $A_{\mathrm{o}}={ }_{3}\langle\cos v\rangle$ is obtained, in principle, from quantum calculations. Surprisingly, $\langle\cos v\rangle$ and thus $A_{\circ}$ are reproduced to

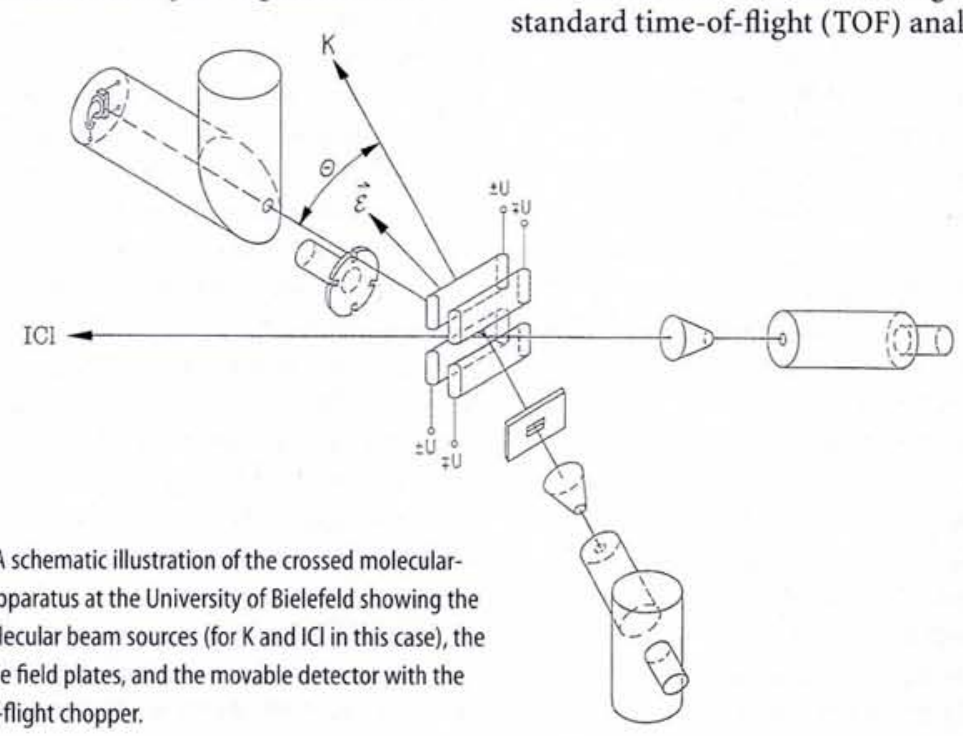

Fig. 3. A schematic illustration of the crossed molecularbeam apparatus at the University of Bielefeld showing the two molecular beam sources (for $\mathrm{K}$ and ICl in this case), the rotatable field plates, and the movable detector with the time-of-flight chopper. within a few percent by the classical Langevin-Debye relation:

$$
\langle\cos v\rangle \cong D \varepsilon / 3 \mathrm{k} T_{\text {rot }}
$$

\section{Reactive Scattering}

Properties of pendular states, in particular the localization near $v=0$ of the lowest states, have recently been probed using collisions and spectroscopic measurements. The former employs the brute force technique to investigate steric effects in reactive collisions, i.e., the effect of the orientation of the molecular axis with respect to the relative velocity of the reactants. The first system to be considered [10] was the reaction $\mathrm{K}+\mathrm{CH}_{3} \mathrm{I} \rightarrow$ $\mathrm{KI}+\mathrm{CH}_{3}$ which had been studied many years ago using a technique based on hexapole focussing. Subsequently, van Leuken et al. investigated the analogous reaction $\mathrm{K}+\mathrm{CH}_{3} \mathrm{Br} \rightarrow \mathrm{KBr}+\mathrm{CH}_{3}$ for which only product angular distributions at thermal energies, but no steric data, were known. The unique feature of the brute-force technique, namely its applicability to all type of polar molecules in $\Sigma$ states, was then exploited in a study of the steric effects involved in the reaction $\mathrm{K}+\mathrm{ICl} \rightarrow \mathrm{KCl}+\mathrm{I}, \mathrm{KI}+\mathrm{Cl}[8]$.

The experimental arrangement common to all three experiments is shown in Fig. 3. Two supersonic beams of seeded reagents intersect at right angles. The products formed in the intersection volume enter a detector chamber were they are ionized on a hot rhenium surface. The detector can be rotated around the intersection volume in the plane containing the two molecular beams in order to measure the angular distributions of products. Product velocity distributions are measured using a standard time-of-flight (TOF) analysis 


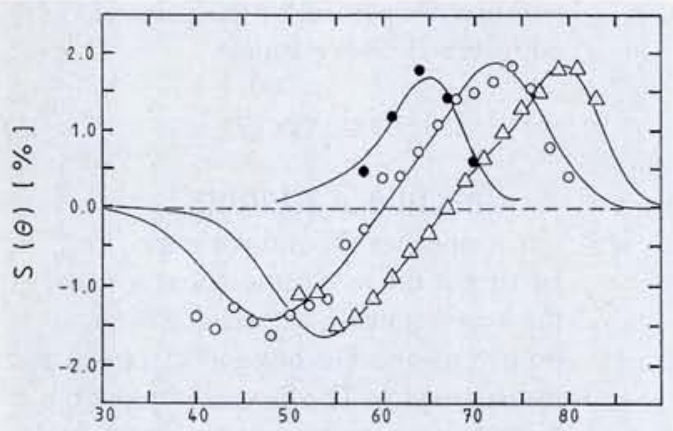

a

LAB scattering angle, $\theta$ [deg]

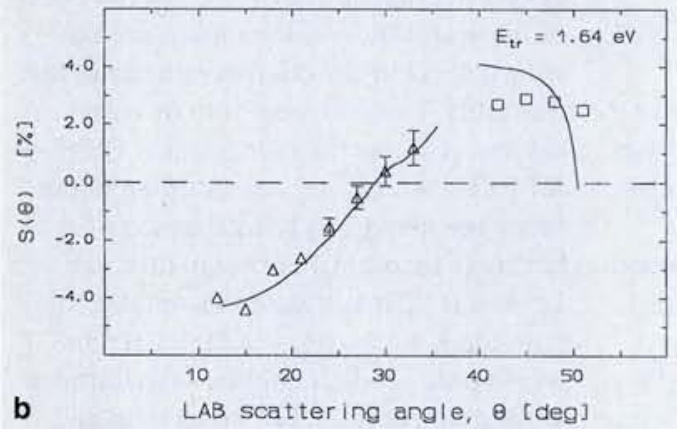

b

LAB scattering angle, 8 [deg

based on a rapidly rotating slit chopper wheel.

Information about product velocities is crucial because both the non-reactively scattered $\mathrm{K}$ atoms and the products $\mathrm{KX}$ ( $X$ denotes a halogen atom) are equally well detected. But, as atoms and products usually travel with drastically different velocities, the TOF distributions can be used to separate out these two contributions to the ion signal.

The orientation field surrounding the intersection volume is generated by two parallel plates which are positioned perpendicular to the plane of the two molecular beams. They each have a slit in this plane to let the beams and the scattered products pass freely. By applying potentials $\pm U$, a field $\varepsilon$ is generated which can be rotated in the scattering plane by rotating the electrode assembly. Reversion of the field direction is achieved by switching the sign of the potentials.

Assuming a Maxwell-Boltzmann rotational distribution, the distribution of the molecular axis is described by Eq. (1). Quantum calculations provide the following values of the anisotropy parameter $A_{\mathrm{o}}$ : $\mathrm{CH}_{3} \mathrm{I}\left(|\varepsilon|=1.6 \mathrm{MV} / \mathrm{m}, T_{\text {rot }}=31 \mathrm{~K}\right): 0.0189$ $\mathrm{CH}_{3} \mathrm{Br}\left(|\varepsilon|=1.88 \mathrm{MV} / \mathrm{m}, T_{\text {rot }}=25 \mathrm{~K}\right): 0.036$ ICI $\left(|\varepsilon|=2.0 \mathrm{MV} / \mathrm{m}, T_{\text {rot }}=14 \mathrm{~K}\right): 0.045$, where the depolarizing influence of nuclear spins is neglected, in agreement with recent calculations which confirm that the decoupling of nuclear spins and rotational angular momentum is essentially complete for such fields [9].
Fig. 4. The velocity-integrated steric effect. a: For the $\mathrm{K}+\mathrm{CH}_{3} \rightarrow \mathrm{KI}+\mathrm{CH}_{3}$ reaction measured at energies $\mathrm{E}_{\mathrm{tr}}$ of 0.79 (triangles) and $1.24 \mathrm{eV}$ (empty circles), and for the $\mathrm{K}+$ $\mathrm{CH}_{3} \mathrm{Br} \rightarrow \mathrm{KBr}+\mathrm{CH}_{3}$ reaction measured at 1.3 $\mathrm{eV}$ (points). The curves are best-fit simulations based on the DIPR model. $\Theta_{\text {LAB }}=$ 0 corresponds to the $K$ beam, $\Theta_{L A B}=90^{\circ}$ to the $\mathrm{CH}_{3} \mathrm{X}$ beam, so forward scattering appears on the left part of the diagram, and backward scattering on the right part. b: For the $\mathrm{K}+\mathrm{ICl} \rightarrow \mathrm{KCl}+\mathrm{I}, \mathrm{KI}+\mathrm{Cl}$ reaction at $E_{\mathrm{tt}}=1.64 \mathrm{eV}$ showing the angular distribution of the steric effect for the fast component of $\mathrm{KCI}$ (triangles) and the slow component of $\mathrm{KI}$ (squares). The curves are best-fit simulations based on the DIPR model. where $\Theta_{\perp A B}=0$ corresponds to the $K$ beam, $\Theta_{\triangle A B}=90^{\circ}$ to the ICI beam.

The relative velocity is well defined because the beam velocity distributions and angular profiles are sufficiently narrow. So cylindrical symmetry of the collision is preserved for the simple case where $\varepsilon$ is parallel or anti-parallel to the relative velocity of the colliding reagents. The flux of products $I\left(\Theta_{L A B}\right)$ is detected as a function of the laboratory scattering angle $\Theta_{\mathrm{LAB}}$ for the two opposite directions of the field (denoted + and -). One can then define a differential steric effect by:

$\mathrm{S}\left(\Theta_{\mathrm{LAB}}\right)=$

$\left[I_{+}\left(\Theta_{\mathrm{LAB}}\right)-I_{-}\left(\Theta_{\mathrm{LAB}}\right)\right] /\left[I_{+}\left(\Theta_{\mathrm{L}_{-A B}}\right)+I_{-}\left(\Theta_{\mathrm{LAB}}\right)\right](3)$

A similar quantity can also be defined for product fluxes measured as functions of the product velocity in the laboratory frame. The full analysis of the experimental signals is relatively difficult, with much of the complexity coming from the transformation from the collision centreof-mass frame to the laboratory frame. In simple cases, the scattering angles in these two frames are monotonically related so that it is straightforward to assign a nature to a $\Theta_{\mathrm{LAB}}$-value, i.e., forward, sideways or backward of the scattering event. In our studies, where scattering involves an atom and a molecule, the term forward/ backward refers in all cases to the trajectory of the atomic reactant.

The differential cross-section $\mathrm{d} \sigma / \mathrm{d} \Omega$ for collisions depends of the angle of attack $\gamma$, the angle between the relative velocity and the molecular axis, and one can expand the differential cross-section as a series of Legendre polynomials

$$
\mathrm{d} \sigma(\gamma) / \mathrm{d} \Omega=\sum_{i=0}^{\infty}(\mathrm{d} \sigma / \mathrm{d} \Omega) P_{l}(\cos \gamma) \quad(4) .
$$

The angle of attack equals either the angle $v$ between the dipole and the field or $\pi-v$, depending on the direction of the orientation field (parallel or antiparallel to the relative velocity vector). The measured signal, which is an average over the distribution of molecular axes (Eq. 1), depends only on the terms $l=0$ and $l=1$ in Eq. 4. Finally, the observed steric effect given by Eq. 3 is obviously a linear function of the anisotropy parameter $A_{0}$.

The $\mathrm{K}+\mathrm{CH}_{3} \mathrm{I} \rightarrow \mathrm{KI}+\mathrm{CH}_{3}$ reaction has been investigated by various groups, each of which measured the angular and velocity distributions of the products at various energies (see [11] for references). All data indicated that $\mathrm{KI}$ is scattered with a high probability, in the backward direction in the centre-of-mass frame: this is called a "rebound reaction". Kuntz [10] rationalized the result using the DIPR (direct interaction with product repulsion) model, whose basic assumptions are: (i) that the KI molecules undergo at the instant of reaction an impulse directed essentially along the CI vector, so that the $\mathrm{KI}$ recoil velocity is strongly correlated to this direction; and (ii) that reaction occurs only if $\mathrm{K}$ hits the molecule within a coneof-acceptance centred around the I-end.

Brute-force experiments have been performed at collision energies at relatively high values $(0.79$ and $1.24 \mathrm{eV})$ of the collision energy $E_{\mathrm{tr}}[4]$. The observed velocity integrated differential steric effect, defined by Eq. 3, is summarised in Fig. 4a. The effect is positive at large $\Theta_{\text {lab }}$ angles, where backward scattering represents the dominant contribution to the signal, and negative at small $\Theta_{\text {lab }}$ angles where forward scattering prevails. A simulation of the angle-dependent steric effect provides the $l=1$ term of the expansion (4), which can be quantitatively recovered using the DIPR model. The results are in qualitative agreement with the results of pioneering experiments by Brooks et al. [11] obtained at thermal collision energies.

The measured steric effect for the reaction $\mathrm{K}+\mathrm{CH}_{3} \mathrm{Br} \rightarrow \mathrm{KBr}+\mathrm{CH}_{3}$ is also plotted in Fig. 4a. It is also well represented by the DIPR model assuming slightly different values of the adjustable parameters.

$\mathrm{The} \mathrm{K}+\mathrm{ICl} \rightarrow \mathrm{KCl}+\mathrm{I}, \mathrm{KI}+\mathrm{Cl}$ reaction is one of the well-studied prototypes 
Fig. 5. Spectroscopy of the pendular states of $\mathrm{ICl}$ in its $\mathrm{A}$ and $X$ electronic states. The upper panel shows the experimental spectrum, as a function of the laser frequency, in the vicinity of the $R_{00}$ line of the $A-X, 21-0$ bandhead ( $a$ few lines labelled by an asterisk belong to another band and are not considered). The middle and lower panels give the result of simulations corresponding to the two signs of the dipole moment $D_{A}$ of the A-state (assuming $D_{x}>0$ ), together with an assignment of the main lines. The hyperfine structure provides an unambiguous assignment of the spectrum and the simulation for $D_{A}>0$ reproduces the experimental spectrum.

of a so-called "stripping reaction". We have, for the first time, oriented and used a ' $\Sigma$ molecule to determine the steric properties of reactive scattering [8].

Experiments were carried out at high collision energies $\left(E_{\mathrm{tr}}=1.61\right.$ and $\left.3.0 \mathrm{eV}\right)$ and the angular and velocity distributions of the products indicated strong forward scattering for both $\mathrm{KCl}$ and $\mathrm{KI}$. The main product is $\mathrm{KCl}$ but, in contrast to earlier studies performed at lower energies, a significant amount of $\mathrm{KI}$ is also produced. Another new feature concerns the recoil velocity distribution of $\mathrm{KCI}$; it exhibits a bimodal distribution owing to the presence of two different pathways for product formation. The high-speed $\mathrm{KCl}$ product exhibits a marked negative orientation effect, while no significant effect is observed for the slow product.

Fig. $4 \mathrm{~b}$ shows the velocity integrated steric effect of the high-speed $\mathrm{KCl}$ product. $S\left(\Theta_{\text {lab }}\right)$ stays essentially negative over the angular range of significant product flux, implying that $\mathrm{KCI}$ is preferentially formed if the atom approaches the I-end of the molecule. On the other hand, $S\left(\Theta_{\text {lab }}\right)$ for KI is positive so the formation of $\mathrm{KI}$ requires approaches towards the $\mathrm{Cl}$ end. The solid lines in Fig. $4 \mathrm{~b}$ are calculated on the basis of the DIPR model, and they essentially provide a quantitative fit to the experimental data. The reason the wrong ends of the molecule are preferred is unclear.

\section{Pendular State Spectroscopy}

The concept of pendular states is very fruitful in spectroscopy. Obviously, the Stark effect on molecular spectra has been studied for a long time, and one could fear that pendular states would merely represent a rewording of the same ideas.

However, this turns out not to be the case and the pendular description can be used to understand prominent features of molecular spectra, especially at high fields.

When the ratio $\omega=D \varepsilon / B$ is made very large, many rotational levels are transformed into pendular levels. The $\mathrm{P}$

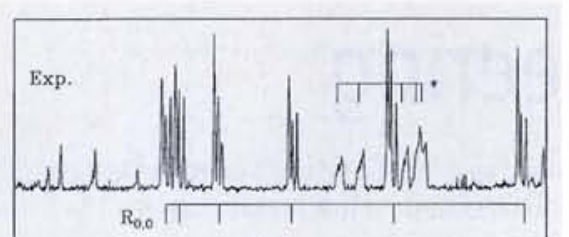

part of the experimental spectrum as well as simulated spectra for the two possible relative signs of the dipoles $D_{\mathrm{A}}$ and $D_{\mathrm{X}}$. The $R_{\circ o}$ line (defined in [14]) connects localized pendular states and its intensity is very sensitive to this sign. The results show unambiguously that the dipole moments of the $\mathrm{A}$ and $\mathrm{X}$ states have the same sign. This conclusion is supported by elementary quantum chemistry arguments and has been demonstrated experimentally elsewhere [15].

\section{Future Developments}

Trying to describe the future of a field is always difficult. One can make a comparison: the manipulation of the position or velocity of atoms by laser techniques is having a great impact on atomic physics. We have considered here the manipulation of the rotational degree of freedom of molecules by an electric field, which can be generalized to magnetic or laser fields, noting however that they unfortunately only align molecules (although their effects may be stronger). The exciting feature is that quantization has a pronounced effect on molecular rotation (as opposed to a weak effect on atomic position). This quantization, which makes the analysis much more difficult, possibly yields more interesting insight.

\section{References}

[1] K.H. Kramer \& R.B. Bernstein,

J. Chem. Phys. 42 (1965) $76 \%$.

[2] S. Stolte, Ber. Bunsenges. Phys. Chem.

86 (1982) 413.

[3] P.R. Brooks, Science 11 (1976) 193.

[4] H.J. Loesch \& A. Remscheid,

J. Chem. Phys. 93 (1990) 4779;

J. Phys. Chem. 95 (1991) 8194.

[5] R. Friedrich \& D.R. Herschbach, Nature 353 (1991) 412.

[6] K. von Meyenn, Z. Phys. 231 (1970) 154.

[7] J. Bulthuis et al., Chem. Phys. Lett. 222

(1994) 378.

[8] H. J. Loesch \& J. Moller, J. Chem. Phys. 97 (1992) 9016; J. Phys. Chem. 97 (1993) 2158.

[9] Loison J.C. et al., J. Phys. Chem. 99 (1995) 13591.

[10] P.J. Kuntz, Mol. Phys. 23 (1972) 1035.

[11] G. Marcelin \& P.R. Brooks, J. Am. Chem.

Soc. 97 (1975) 1710.

[12] P.A. Block, E.J. Bohac \& R.E. Miller,

Phys. Rev. Lett. 68 (1992) 1303.

[13] B. Friedrich et al., J. Chem. Soc. Faraday 89 (1993) 1539.

[14] A. Durand, J.C. Loison \& J. Vigué,

J. Chem. Phys. 101 (1994) 3514.

[15] B. Friedrich, A. Slenczka \& D.R.

Herschbach, Chem. Phys. Lett. 221 (1994) 333. 